\title{
Prepregnancy Physical Activity in relation to Offspring Birth Weight: A Prospective Population-Based Study in Norway-The HUNT Study
}

\author{
Silje Krogsgaard, Sigridur L. Gudmundsdottir, and Tom I. L. Nilsen \\ Department of Human Movement Science, Norwegian University of Science and Technology, 7491 Trondheim, Norway \\ Correspondence should be addressed to Tom I. L. Nilsen; tom.nilsen@svt.ntnu.no
}

Received 12 October 2012; Revised 7 December 2012; Accepted 10 January 2013

Academic Editor: Michelle F. Mottola

Copyright (c) 2013 Silje Krogsgaard et al. This is an open access article distributed under the Creative Commons Attribution License, which permits unrestricted use, distribution, and reproduction in any medium, provided the original work is properly cited.

Background. The objective was to examine the association between prepregnancy physical exercise and offspring birth weight and to assess the combined association of pre-pregnancy body mass index (BMI) and physical exercise on birth weight. Methods. The study included 2,026 women aged 20-39 years participating in the Norwegian HUNT study and linked with the Medical Birth Registry. We calculated mean differences in birth weight and odds ratios (ORs) for a macrosomic infant (i.e., birth weight $>4000 \mathrm{~g}$ ) using linear and logistic regression analysis. Results. There was no clear association between leisure time physical exercise and mean birth weight. Women who reported no exercise had reduced risk of a macrosomic infant (OR, 0.6; 95\% confidence interval (CI), 0.4-0.9) compared to women with a high exercise level. Overweight (BMI $\geq 25.0 \mathrm{~kg} / \mathrm{m}^{2}$ ) was associated with an OR of 1.9 (95\% CI, 1.2-2.9) for a macrosomic infant among women who reported low exercise levels, whereas the OR was 1.2 (95\% CI, 0.8-1.8) among women with higher exercise levels. Conclusion. There was some evidence that women who reported no exercise before pregnancy had lower risk for a macrosomic infant than women who exercised. Pre-pregnancy BMI was positively associated with birth weight and risk of macrosomia but only among the least active women.

\section{Introduction}

The proportion of women giving birth to large infants has increased around the world $[1,2]$, most likely because of the rising rates of maternal overweight and obesity [3-7]. Whereas consequences of low birth weight may include infant mortality and morbidity [8], high birth weight has been related to increased risk for caesarean section, chorioamnionitis, fourth degree perinatal lacerations, postpartum haemorrhage, shoulder dystocia [9-11], and low Apgar score [12]. Additionally, high birth weight has been positively associated with obesity [13] and type 2 diabetes [14] in adulthood.

Previous studies have reported that physical activity in pregnancy is related to foetal growth rate and birth weight $[15,16]$, and that physically active women have a reduced risk of delivering a large infant $[17,18]$, possibly by increased insulin sensitivity [6]. However, not all studies have reported consistent inverse associations between physical activity in pregnancy and birth weight [19-22]. Although women who exercise regularly before pregnancy are more likely to continue to exercise during pregnancy [23-25], few studies have examined the associations between prepregnancy physical activity and birthweight, and the results have been inconsistent $[18,20,26,27]$.

In this prospective study in Norway, we have utilized data on women participating in a population-based health study linked with information from the Medical Birth Registry to study the association between maternal pre-pregnancy physical exercise and offspring birth weight. Additionally, we explored the combined association of pre-pregnancy body mass index and physical exercise on birth weight.

\section{Materials and Methods}

2.1. Study Population. The Nord-Trøndelag health study (The HUNT study) is a large population-based health study conducted in the county of Nord-Trøndelag, Norway. The HUNT 
TABLE 1: Characteristics of the study population $(N=2,026)$ according to total leisure time physical exercise.

\begin{tabular}{|c|c|c|c|c|}
\hline \multirow{2}{*}{ Characteristic } & \multicolumn{4}{|c|}{ Total physical exercise level $^{\mathrm{a}}$} \\
\hline & No activity & Low & Medium & High \\
\hline No participants (\% of total) & $147(7.3)$ & $647(31.9)$ & $641(31.6)$ & $591(29.2)$ \\
\hline Mean age at baseline, $y$ & 26.8 & 27.2 & 26.7 & 26.6 \\
\hline Mean body mass index, $\mathrm{kg} / \mathrm{m}^{2}$ & 22.4 & 22.3 & 22.5 & 22.4 \\
\hline Parity (\% primiparous) & 20.4 & 28.2 & 28.5 & 40.6 \\
\hline Smoking (\% current smoking) & 53.1 & 44.0 & 38.8 & 31.3 \\
\hline Education (\% college/university) & 8.1 & 16.1 & 20.6 & 25.4 \\
\hline Alcohol (\% not drinking last 2 weeks) & 49.7 & 45.7 & 46.0 & 43.1 \\
\hline Marital status (\% married) & 53.1 & 55.6 & 49.5 & 46.7 \\
\hline
\end{tabular}

aased on a summary score of frequency, duration, and intensity of exercise.

study is a collaboration between HUNT Research Centre (Faculty of Medicine, Norwegian University of Science and Technology NTNU), Nord-Trøndelag County Council, Central Norway Health Authority, and the Norwegian Institute of Public Health. It constitutes three consecutive cross-sectional waves; the first was conducted in 1984-1986 (HUNT 1), the second in 1995-1997 (HUNT 2), and the third in 20062008 (HUNT 3). For the purpose of the present study, we have used information from the first wave (HUNT 1). In HUNT 1, 87,285 persons aged $\geq 20$ years were invited to participate, and 77,216 (88.5\%) accepted the invitation, filled in questionnaires, and attended a clinical examination $(37,826$ men and 39,390 women). A more detailed description of participation, method, and procedures of the HUNT study can be found elsewhere [28].

For the purpose of the current study, we first selected all 3,739 women aged 20-39 years at participation in HUNT 1 (1984-1986) who gave birth to at least one child during a five year period after participation. Of these 3,739 women, we excluded 232 women due to pre- (<37 weeks) or postterm (>44 weeks) delivery, 32 women with multiple births, 10 women with gestational diabetes, 118 women with preeclampsia, 831 women without information of physical activity, body mass index, or gestational age, and 490 women who gave birth within 10 months after participation (i.e., possibly pregnant at the time of participation). This left 2,026 women available for statistical analysis.

2.2. Study Variables. Information on the offspring was obtained by a linkage to the Medical Birth Registry of Norway. These data were obtained for the first child born during five years after participation in HUNT. The main outcome variable was newborn birth weight, measured in grams (g), first analyzed as a continuous variable and then as a dichotomized variable using 4,000 $\mathrm{g}$ as cutoff. Macrosomia was defined as birth weight at or above 4,000 g [29].

Leisure time physical exercise was assessed using three questions. In the first question, the participants were asked to report how many exercise sessions (e.g., walking, skiing, swimming, or other sports) they usually had during a week, with five response options $(0,<1,1,2-3$, and $\geq 4$ times; coded 1-5). If the participants reported exercising at least once a week, they were also asked to report the average duration $(<15,15-30,31-60$, and $>60$ minutes; coded $1-4)$ and intensity (light, moderate, and to exhaustion; coded 1-3) of the activity. Among participants who reported exercising at least once a week, a summary score of frequency, duration, and intensity was calculated according to the following equation: $1 / 5 *$ frequency $+1 / 4 *$ duration $+1 / 3 *$ intensity. This procedure intended to give equal weight to each component of physical activity and resulted in a maximum score of 1.0 for each of the three components of the summary score. The median score value of 1.97 (range, 1.2-3.0) was then used as a cut-off to classify women into two categories of score values ( \pm median). This information was used to construct a variable of total physical exercise with four unique categories: (1) no activity, (2) low activity (<1 session per week), (3) medium activity ( $<$ median score value), and (4) high activity ( $\geq$ median score value) [30].

Body mass index (BMI) was computed from standardized measures as weight divided by the squared value of height $\left(\mathrm{kg} / \mathrm{m}^{2}\right)$ and categorized into two groups: BMI $<25.0 \mathrm{~kg} / \mathrm{m}^{2}$ and $\mathrm{BMI} \geq 25.0 \mathrm{~kg} / \mathrm{m}^{2}$ (i.e., overweight or obese) [31].

2.3. Statistical Analysis. We used linear regression to analyze the association between measures of leisure time physical exercise and mean birth weight. We also calculated odds ratios (OR) for having a macrosomic infant $(\geq 4,000 \mathrm{~g})$ in different categories of leisure time physical exercise using logistic regression. Precision of the estimated associations was assessed by a 95\% confidence interval (CI). Women who reported the highest activity level were used as the reference category in all analysis. The following variables were considered as potential confounders in the analysis; age (2024, 25-29, 30-34, and 35-39 years), smoking (never, former, and current), frequency of alcohol consumption during the past 2 weeks (none, 1-4 times, $\geq 5$ times, abstainer, and unknown), education ( $<10,10-12,>12$ years, and unknown), marital status (unmarried, married, and previously married), and parity (primiparous, 1-2 children, and 3-6 children). Covariates were removed from the model if there was no meaningful difference between adjusted and unadjusted estimates. All estimates were adjusted for maternal age, smoking, and parity. Tests for trend across categories of leisure time physical exercise were conducted by treating the categories as an ordinal variable in the regression model. 
TABLE 2: Maternal pre-pregnancy leisure time physical exercise and mean offspring birth weight from linear regression analyses.

\begin{tabular}{|c|c|c|c|c|c|}
\hline Physical exercise & No. of persons & Mean birth weight $(\mathrm{g})$ & Crude difference & Adjusted $^{\text {a }}$ difference $(95 \%$ CI) & $P$ trend $^{\mathrm{b}}$ \\
\hline \multicolumn{6}{|l|}{ Sessions per week } \\
\hline None & 147 & 3589.0 & -25.2 & $-26.9(-137.9$ to 84.2$)$ & \\
\hline$<1$ & 647 & 3632.5 & 18.3 & $21.7(-64.8$ to 108.3$)$ & \\
\hline 1 & 591 & 3639.7 & 25.5 & $31.0(-56.1$ to 118.1$)$ & \\
\hline $2-3$ & 491 & 3590.4 & -23.7 & $-24.4(-113.2$ to 64.4$)$ & \\
\hline$\geq 4$ & 150 & 3614.1 & 0.0 & 0.0 (reference) & 0.49 \\
\hline \multicolumn{6}{|l|}{ Total exercise ${ }^{c}$} \\
\hline No activity & 147 & 3589.0 & -38.9 & $-53.0(-141.8$ to 35.8$)$ & \\
\hline Low & 647 & 3632.5 & 4.6 & $-3.5(-58.3$ to 51.4$)$ & \\
\hline Medium & 641 & 3606.9 & -21.0 & $-36.9(-91.5$ to 17.8$)$ & \\
\hline High & 591 & 3627.9 & 0.0 & 0.0 (reference) & 0.56 \\
\hline
\end{tabular}

CI: confidence interval.

${ }^{a}$ Adjusted for maternal age (20-24, 25-29, 30-34, and 35-39 years), smoking (never, former, current, and unknown), and parity (primiparous, 1-2 children, and 3-6 children).

${ }^{\mathrm{b}} P$ value from trend test when categories were entered as an ordinal variable in the regression model.

${ }^{\mathrm{c}}$ Based on a summary score of frequency, duration, and intensity of exercise.

Since maternal BMI could be both an effect modifier and on the causal pathway between exercise and birth weight, BMI was not included as a confounder in the primary analyses. However, additional analysis was conducted for the combined associations of prepregnancy BMI and total leisure time physical exercise in relation to birth weight, using linear and logistic regression as described earlier. We also included a product term of $\mathrm{BMI}\left(<25\right.$ versus $\left.\geq 25 \mathrm{~kg} / \mathrm{m}^{2}\right)$ and exercise level (no or low activity versus medium or high activity) to assess possible interaction between the two variables and as well as stratified the analyses of physical exercise on the two BMI groups.

All statistical analyses were performed using the statistical software SPSS for Windows, version 17.0.

The study was approved by the Regional Committee for Ethics in Medical Research. All eligible participants received a written invitation with information about the study, and all participants gave their consent by filling in and returning the first questionnaire that was mailed together with the invitation.

\section{Results}

Descriptive characteristics of the study population are presented in Table 1. Mean baseline maternal age among the 2,026 women in the study was 26.9 years, whereas mean birth weight of their offspring was $3,620 \mathrm{~g}(\mathrm{SD}, 502)$, and a total of $416(20.5 \%)$ newborns weighed $4,000 \mathrm{~g}$ or more (i.e., had macrosomia).

Table 2 presents results from linear regression showing that there was no clear association between maternal leisure time physical exercise and mean birth weight of their offspring, neither in relation to number of exercise sessions per week ( $P$ trend, 0.49 ) nor in relation to total amount of exercise ( $P$ trend, 0.56 ). Correspondingly, results from logistic regression presented in Table 3 provide no consistent evidence for an association between maternal
TABLE 3: Odds ratio (OR) from logistic regression for giving birth to a marcosomic infant (i.e., birth weight $>4000 \mathrm{~g}$ ) in association with maternal pre-pregnancy leisure time physical exercise.

\begin{tabular}{lccccc}
\hline Physical exercise & $\begin{array}{c}\text { No. of } \\
\text { persons }\end{array}$ & $\begin{array}{c}\text { No. } \\
\text { of } \\
\text { cases }\end{array}$ & $\begin{array}{c}\text { Crude } \\
\text { OR }\end{array}$ & $\begin{array}{c}\text { Adjusted }^{\mathrm{a}} \text { OR } \\
(95 \% \mathrm{CI})\end{array}$ & $P$ trend $^{\mathrm{b}}$ \\
\hline Sessions per week & & & & & \\
$\quad$ None & 147 & 20 & 0.7 & $0.7(0.4-1.3)$ & \\
$<1$ & 647 & 148 & 1.3 & $1.3(0.8-2.1)$ & \\
1 & 591 & 122 & 1.1 & $1.2(0.7-1.8)$ & \\
$2-3$ & 491 & 98 & 1.2 & $1.1(0.7-1.8)$ & \\
$\geq 4$ & 150 & 28 & 1.0 & $1.0($ reference) & 0.89 \\
Total exercise & & & & & \\
No activity & 147 & 20 & 0.6 & $0.6(0.4-0.9)$ & \\
Low & 647 & 148 & 1.2 & $1.1(0.9-1.5)$ & \\
Medium & 641 & 128 & 1.0 & $0.9(0.7-1.2)$ & \\
High & 591 & 120 & 1.0 & $1.0($ reference) & 0.65 \\
\hline
\end{tabular}

CI: confidence interval.

${ }^{a}$ Adjusted for maternal age (20-24, 25-29, 30-34, and 35-39 years), smoking (never, former, current, and unknown), and parity (primiparous, 1-2 children, and 3-6 children).

${ }^{\mathrm{b}} P$ value from trend test when categories were entered as an ordinal variable in the regression model.

${ }^{c}$ Based on a summary score of frequency, duration, and intensity of exercise.

physical exercise and risk of a macrosomic infant. However, women who reported being inactive before pregnancy had a lower risk of giving birth to an infant with excessive birth weight (OR, 0.6; 95\% CI, 0.4-0.9) compared to women with a high total exercise level (Table 3 ).

The combined association of pre-pregnancy BMI and leisure time physical exercise in relation to birth weight is shown in Table 4 . Women who were overweight (BMI $\geq$ $25.0 \mathrm{~kg} / \mathrm{m}^{2}$ ) before pregnancy and reported no or low leisure time physical exercise gave birth to infants with significantly 
TABLE 4: Maternal pre-pregnancy body mass index (BMI) and leisure time physical exercise related to mean offspring birth weight from linear regression and odds ratio (OR) from logistic regression for a macrosomic infant (i.e., birth weight $>4000 \mathrm{~g}$ ).

\begin{tabular}{|c|c|c|c|c|c|c|}
\hline $\begin{array}{l}\text { Combined categories of } \\
\text { BMI and exercise } \mathrm{e}^{\mathrm{a}}\end{array}$ & $\begin{array}{l}\text { No. of } \\
\text { persons }\end{array}$ & $\begin{array}{l}\text { Crude mean } \\
\text { difference }\end{array}$ & $\begin{array}{c}\text { Adjusted }^{\mathrm{b}} \text { mean } \\
\text { difference }(95 \% \mathrm{CI})\end{array}$ & $\begin{array}{l}\text { No. of cases } \\
(\mathrm{BW}>4000 \mathrm{~g})\end{array}$ & Crude OR & $\begin{array}{l}\text { Adjusted }^{\mathrm{b}} \text { OR } \\
\quad(95 \% \mathrm{CI})\end{array}$ \\
\hline $\begin{array}{l}<25 \mathrm{~kg} / \mathrm{m}^{2} \text { and } \\
\text { medium } / \text { high level }\end{array}$ & 1,046 & 0.0 & 0.0 (reference) & 206 & 1.0 & 1.0 (reference) \\
\hline$<25 \mathrm{~kg} / \mathrm{m}^{2}$ and no/low level & 676 & -8.6 & $-8.4(-55.6$ to 38.8$)$ & 131 & 1.0 & $1.0(0.8-1.2)$ \\
\hline $\begin{array}{l}\geq 25 \mathrm{~kg} / \mathrm{m}^{2} \text { and } \\
\text { medium } / \text { high level }\end{array}$ & 186 & 29.8 & $33.1(-42.5$ to 108.7$)$ & 42 & 1.2 & $1.2(0.8-1.8)$ \\
\hline$\geq 25 \mathrm{~kg} / \mathrm{m}^{2}$ and no/low level & 118 & 129.7 & $134.1(41.0$ to 227.3$)$ & 37 & 1.9 & $1.9(1.2-2.9)$ \\
\hline
\end{tabular}

CI: confidence interval; BW: birth weight.

${ }^{a}$ Based on a summary score of frequency, duration, and intensity of exercise.

${ }^{\mathrm{b}}$ Adjusted for maternal age (20-24, 25-29, 30-34, and 35-39 years), smoking (never, former, current, and unknown), and parity (primiparous, 1-2 children, and 3-6 children).

higher mean birth weight (134 g; 95\% CI, 41.0-227.3) and had a higher risk for a macrosomic infant (OR, 1.9; 95\% CI, 1.2$2.9)$, compared to women with BMI $<25.0 \mathrm{~kg} / \mathrm{m}^{2}$ who had a medium or high exercise level. In additional analysis stratified by body mass index, overweight women (BMI $\geq 25.0 \mathrm{~kg} / \mathrm{m}^{2}$ ) who reported no or low exercise levels had significantly higher offspring birth weight (132 g; 95\% CI, 20.4-243.7) and higher odds ratio for a macrosomic infant (OR, 2.0; 95\% CI, 1.1-3.5) than women who reported a medium or high exercise level (data not shown). However, there was no statistically significant interaction between BMI and total exercise level $(P=0.08)$.

\section{Discussion}

In this large prospective study of Norwegian women, we found no clear association between reported leisure time physical exercise level before pregnancy and offspring birth weight. There was some evidence that inactive women had a slightly lower likelihood of giving birth to a child with excessive birth weight than more physically active women, but the small numbers of inactive women call for a cautious interpretation. Analysis of the combined association of BMI and exercise on birth weight showed that women with a BMI $\geq 25.0 \mathrm{~kg} / \mathrm{m}^{2}$ gave birth to infants with significantly higher birth weight than women with a BMI $<25.0 \mathrm{~kg} / \mathrm{m}^{2}$, but only if they also reported no or low levels of physical exercise. This could suggest that physical exercise to some extent reduces the effect of maternal adiposity on offspring birth weight.

The suggestive evidence that women who were inactive before pregnancy had lower risk for delivering a macrosomic infant is contradictory to some previous studies. Voldner et al. [20] reported that inactive women (defined as $<1 \mathrm{~h}$ per week) had almost a threefold higher odds ratio for fetal macrosomia than physically active women ( $>1 \mathrm{~h}$ per week). However, another Norwegian study found no association between frequency of regular exercise before pregnancy and offspring with excessive birth weight ( $\geq 90$ th percentile) [18]. In the present study, those who were classified as inactive reported never engaging in physical exercise, and these women could be more extremely sedentary than inactive women in other studies. Studies have shown that a sedentary lifestyle in pregnancy is associated with lower birth weight [32] and an increased risk of a very low birth weight infant [16]. It has been observed that mothers of very low birth weight infants were less likely to be physically active during their pregnancy [33]. It has been suggested that both excessive and insufficient physical activities in pregnancy are related to an inadequate fetal growth [34], although some women might be advised to be inactive and at rest to reduce the risk of adverse pregnancy outcomes.

Unlike the present study, some previous studies have shown inverse associations between maternal pre-pregnancy exercise behaviors and offspring birth weight or risk of having excess weight $[17,18]$, although the results are not entirely consistent [21]. A recent study of leisure time physical activity during pregnancy is more in agreement with the results of the present study. Hegaard et al. [22] found no association with mean birth weight or the risk of giving birth to low $(<2,500 \mathrm{~g})$ or high $(\geq 4,500 \mathrm{~g})$ birth weight infants. The inconsistent results in these studies could be due to different measures of physical activity, in addition to the possibility for chance findings in the smaller studies.

There is growing evidence that overweight or obesity before pregnancy is a risk factor for macrosomia $[3,12,35,36]$. The results from the present study suggest that the effects of maternal pre-pregnancy overweight were associated with higher birth weight only among women who reported no or low level of activity. This is contradictory to the findings by Löf et al. [27] who showed that a high pre-pregnancy activity level did not reduce the risk of high birth weight infants in women who were overweight or gained much weight in pregnancy. Nevertheless, physical activity may improve maternal weight control, both before and during pregnancy $[37,38]$.

The strengths of the present study include the prospective design, the large sample size of women reporting physical activity before pregnancy, and the standardized measures of size at birth obtained from the Medical Birth Registry of Norway. However, some of the categories of physical exercise (e.g., inactive) suffered from small samples size, and this could result in chance findings. Moreover, as in any observational study, residual confounding due to unmeasured 
(e.g., smoking during pregnancy and gestational weight gain) or unknown factors cannot be ruled out. Since the information on physical activity was self-reported, it could be subject to misclassification, although a validation study has shown acceptable agreement with objective measures [39]. Although pre-pregnancy physical activity has been associated with physical activity level during pregnancy [23-25], it has also been shown that exercise levels decline in pregnancy $[24,25,40,41]$. Unfortunately, our data did not allow us to examine if pre-pregnancy physical exercise was related to the activity level during pregnancy.

\section{Conclusions}

There was no clear association between maternal prepregnancy leisure time physical exercise and offspring birth weight. However, the result may indicate that inactive women gave birth to infants with lower birth weight and had lower risk for delivering an infant with macrosomia. The results also show that high maternal pre-pregnancy BMI was associated with higher mean birth weight and increased risk of macrosomia in offspring of physically inactive women, whereas pre-pregnancy BMI was not associated with birth weight and risk of macrosomia among more active women.

\section{Conflict of Interests}

The authors declare that they have no conflict of interests.

\section{References}

[1] M. S. Kramer, I. Morin, H. Yang et al., "Why are babies getting bigger? Temporal trends in fetal growth and its determinants," Journal of Pediatrics, vol. 141, no. 4, pp. 538-542, 2002.

[2] J. Ørskou, U. Kesmodel, T. B. Henriksen, and N. J. Secher, "An increasing proportion of infants weigh more than 4000 grams at birth," Acta Obstetricia et Gynecologica Scandinavica, vol. 80, no. 10, pp. 931-936, 2001.

[3] S. Bhattacharya, D. M. Campbell, W. A. Liston, and S. Bhattacharya, "Effect of body mass index on pregnancy outcomes in nulliparous women delivering singleton babies," BMC Public Health, vol. 7, article 168, 2007.

[4] H. M. Ehrenberg, L. Dierker, C. Milluzzi, and B. M. Mercer, "Prevalence of maternal obesity in an urban center," American Journal of Obstetrics and Gynecology, vol. 187, no. 5, pp. 11891193, 2002.

[5] S. Phelan, C. Hart, M. Phipps et al., "Maternal behaviors during pregnancy impact offspring obesity risk," Experimental Diabetes Research, vol. 2011, Article ID 985139, 9 pages, 2011.

[6] S. M. Nelson, P. Matthews, and L. Poston, "Maternal metabolism and obesity: modifiable determinants of pregnancy outcome," Human Reproduction Update, vol. 16, no. 3, pp. 255-275, 2010.

[7] http://www.who.int/mediacentre/factsheets/fs311/en/index. html

[8] M. S. Kramer, "The epidemiology of adverse pregnancy outcomes: an overview," Journal of Nutrition, vol. 133, no. 5, supplement 2, pp. 1592S-1596S, 2003.

[9] T. Henriksen, "The macrosomic fetus: a challenge in current obstetrics," Acta Obstetricia et Gynecologica Scandinavica, vol. 87, no. 2, pp. 134-145, 2008.
[10] N. E. Stotland, A. B. Caughey, E. M. Breed, and G. J. Escobar, "Risk factors and obstetric complications associated with macrosomia," International Journal of Gynecology and Obstetrics, vol. 87, no. 3, pp. 220-226, 2004.

[11] N. Voldner, K. F. Frøslie, L. A. H. Haakstad, K. Bø, and T. Henriksen, "Birth complications, overweight, and physical inactivity," Acta Obstetricia et Gynecologica Scandinavica, vol. 88, no. 5, pp. 550-555, 2009.

[12] M. C. Jolly, N. J. Sebire, J. P. Harris, L. Regan, and S. Robinson, "Risk factors for macrosomia and its clinical consequences: a study of 350,311 pregnancies," European Journal of Obstetrics Gynecology and Reproductive Biology, vol. 111, no. 1, pp. 9-14, 2003.

[13] W. McGuire, L. Dyson, and M. Renfrew, "Maternal obesity: consequences for children, challenges for clinicians and carers," Seminars in Fetal and Neonatal Medicine, vol. 15, no. 2, pp. 108$112,2010$.

[14] T. Harder, E. Rodekamp, K. Schellong, J. W. Dudenhausen, and A. Plagemann, "Birth weight and subsequent risk of type 2 diabetes: a meta-analysis," American Journal of Epidemiology, vol. 165, no. 8, pp. 849-857, 2007.

[15] J. F. Clapp III, H. Kim, B. Burciu, and B. Lopez, "Beginning regular exercise in early pregnancy: effect on fetoplacental growth," American Journal of Obstetrics and Gynecology, vol. 183, no. 6, pp. 1484-1488, 2000.

[16] J. A. Leiferman and K. R. Evenson, "The effect of regular leisure physical activity on birth outcomes," Maternal and Child Health Journal, vol. 7, no. 1, pp. 59-64, 2003.

[17] B. W. Alderman, H. Zhao, V. L. Holt, D. H. Watts, and S. A. A. Beresford, "Maternal physical activity in pregnancy and infant size for gestational age," Annals of Epidemiology, vol. 8, no. 8, pp. 513-519, 1998.

[18] K. M. Owe, W. Nystad, and K. Bø, "Association between regular exercise and excessive newborn birth weight," Obstetrics and Gynecology, vol. 114, no. 4, pp. 770-776, 2009.

[19] A. M. Z. Jukic, K. R. Evenson, J. L. Daniels, A. H. Herring, A. J. Wilcox, and K. E. Hartmann, "A prospective study of the association between vigorous physical activity during pregnancy and length of gestation and birthweight," Maternal and Child Health Journal, vol. 16, no. 5, pp. 1031-1044, 2012.

[20] N. Voldner, K. F. Frøslie, K. Bø et al., "Modifiable determinants of fetal macrosomia: role of lifestyle-related factors," Acta Obstetricia et Gynecologica Scandinavica, vol. 87, no. 4, pp. 423429, 2008.

[21] M. Juhl, J. Olsen, P. K. Andersen, E. A. Nøhr, and A. M. N. Andersen, "Physical exercise during pregnancy and fetal growth measures: a study within the Danish National Birth Cohort," American Journal of Obstetrics and Gynecology, vol. 202, no. 1, pp. 63.el-63.e8, 2010.

[22] H. K. Hegaard, K. Petersson, M. Hedegaard et al., "Sports and leisure-time physical activity in pregnancy and birth weight: a population-based study," Scandinavian Journal of Medicine and Science in Sports, vol. 20, no. 1, pp. e96-e102, 2010.

[23] L. A. H. Haakstad, N. Voldner, T. Henriksen, and K. Bø, "Why do pregnant women stop exercising in the third trimester?" Acta Obstetricia et Gynecologica Scandinavica, vol. 88, no. 11, pp. 1267-1275, 2009.

[24] K. M. Owe, W. Nystad, and K. Bø, "Correlates of regular exercise during pregnancy: the Norwegian Mother and Child Cohort Study," Scandinavian Journal of Medicine and Science in Sports, vol. 19, no. 5, pp. 637-645, 2009. 
[25] H. K. Hegaard, P. Damm, M. Hedegaard et al., "Sports and leisure time physical activity during pregnancy in nulliparous women," Maternal and Child Health Journal, vol. 15, no. 6, pp. 806-813, 2011.

[26] C. Fleten, H. Stigum, P. Magnus, and W. Nystad, "Exercise during pregnancy, maternal prepregnancy body mass index, and birth weight," Obstetrics and Gynecology, vol. 115, no. 2, part 1, pp. 331-337, 2010.

[27] M. Löf, L. Hilakivi-Clarke, S. Sandin, and E. Weiderpass, "Effects of pre-pregnancy physical activity and maternal BMI on gestational weight gain and birth weight," Acta Obstetricia et Gynecologica Scandinavica, vol. 87, no. 5, pp. 524-530, 2008.

[28] S. Krokstad, A. Langhammer, K. Hveem et al., "Cohort profile: the HUNTStudy, Norway," International Journal of Epidemiology. In press.

[29] S. L. Boulet, G. R. Alexander, H. M. Salihu, and M. Pass, "Macrosomic births in the United States: determinants, outcomes, and proposed grades of risk," American Journal of Obstetrics and Gynecology, vol. 188, no. 5, pp. 1372-1378, 2003.

[30] T. I. L. Nilsen, P. R. Romundstad, and L. J. Vatten, "Recreational physical activity and risk of prostate cancer: a prospective population-based study in Norway (the HUNT study)," International Journal of Cancer, vol. 119, no. 12, pp. 2943-2947, 2006.

[31] http://www.who.int/mediacentre/factsheets/fs311/en/index. html

[32] M. I. Both, M. A. Overvest, M. F. Wildhagen, J. Golding, and H. I. J. Wildschut, "The association of daily physical activity and birth outcome: a population-based cohort study," European Journal of Epidemiology, vol. 25, no. 6, pp. 421-429, 2010.

[33] W. F. Schramm, J. W. Stockbauer, and H. J. Hoffman, "Exercise, employment, other daily activities, and adverse pregnancy outcomes," American Journal of Epidemiology, vol. 143, no. 3, pp. 211-218, 1996.

[34] M. Y. Takito, M. H. Benício, and L. C. Neri, "Physical activity by pregnant women and outcomes for newborns: a systematic review," Revista de saúde pública, vol. 43, no. 6, pp. 1059-1069, 2009.

[35] I. O. Frederick, M. A. Williams, A. E. Sales, D. P. Martin, and M. Killien, "Pre-pregnancy body mass index, gestational weight gain, and other maternal characteristics in relation to infant birth weight," Maternal and Child Health Journal, vol. 12, no. 5, pp. 557-567, 2008.

[36] H. M. Ehrenberg, B. M. Mercer, and P. M. Catalano, "The influence of obesity and diabetes on the prevalence of macrosomia," American Journal of Obstetrics and Gynecology, vol. 191, no. 3, pp. 964-968, 2004.

[37] K. Melzer, Y. Schutz, M. Boulvain, and B. Kayser, "Physical activity and pregnancy: cardiovascular adaptations, recommendations and pregnancy outcomes," Sports Medicine, vol. 40, no. 6, pp. 493-507, 2010.

[38] Z. Sui, R. M. Grivell, and J. M. Dodd, "Antenatal exercise to improve outcomes in overweight or obese women: a systematic review," Acta Obstetricia et Gynecologica Scandinavica, vol. 91, no. 5, pp. 538-545, 2012.

[39] N. Kurtze, V. Rangul, B. E. Hustvedt, and W. D. Flanders, "Reliability and validity of self-reported physical activity in the Nord-Trøndelag Health Study (HUNT 2)," European Journal of Epidemiology, vol. 22, no. 6, pp. 379-387, 2007.

[40] L. A. H. Haakstad, N. Voldner, T. Henriksen, and K. Bø, "Physical activity level and weight gain in a cohort of pregnant Norwegian women," Acta Obstetricia et Gynecologica Scandinavica, vol. 86, no. 5, pp. 559-564, 2007.
[41] D. B. Fell, K. S. Joseph, B. A. Armson, and L. Dodds, "The impact of pregnancy on physical activity level," Maternal and Child Health Journal, vol. 13, no. 5, pp. 597-603, 2009. 


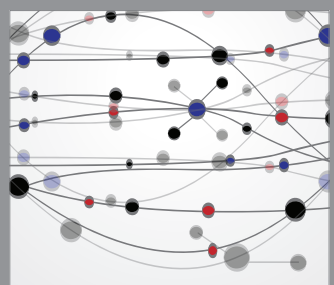

The Scientific World Journal
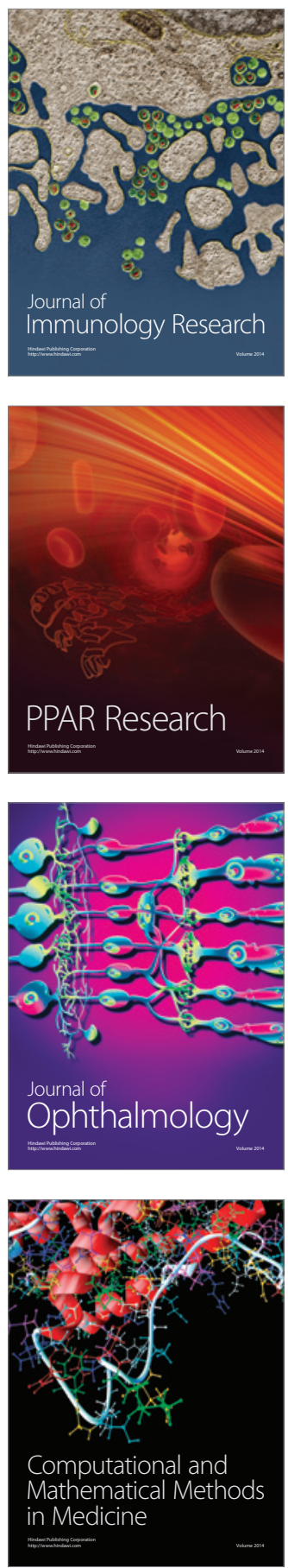

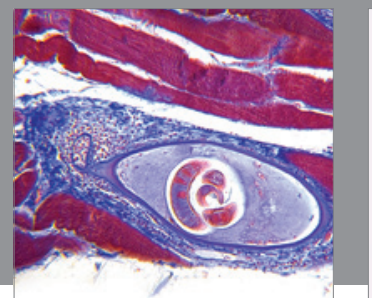

Gastroenterology

Research and Practice
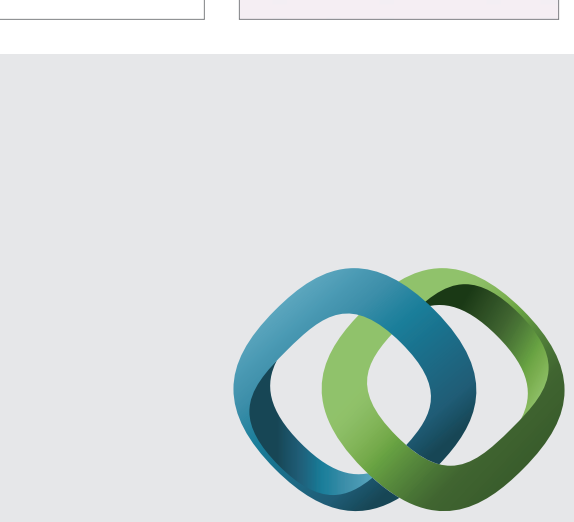

\section{Hindawi}

Submit your manuscripts at

http://www.hindawi.com
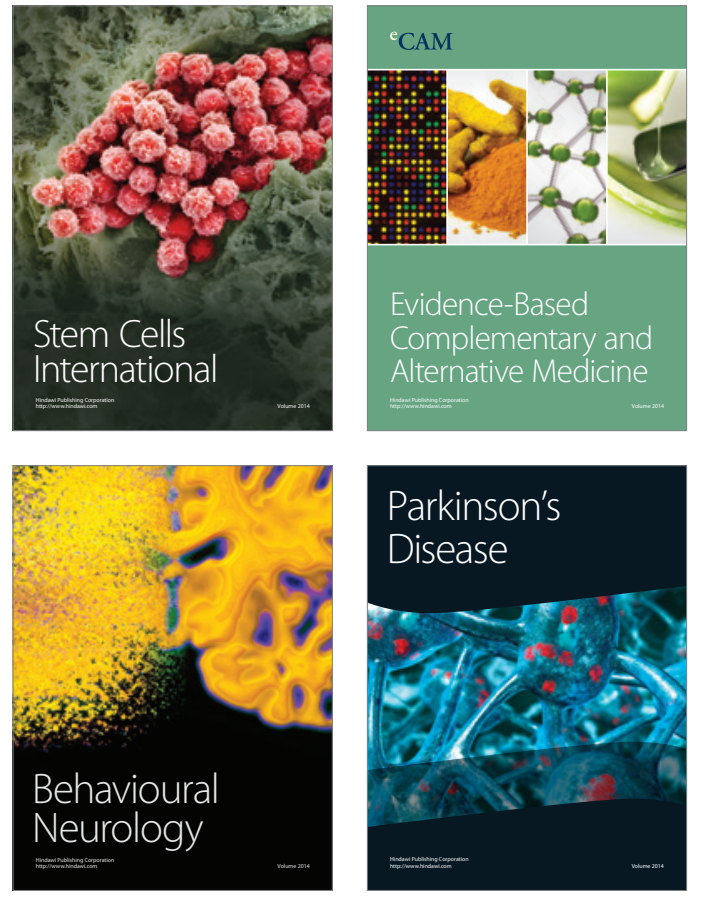
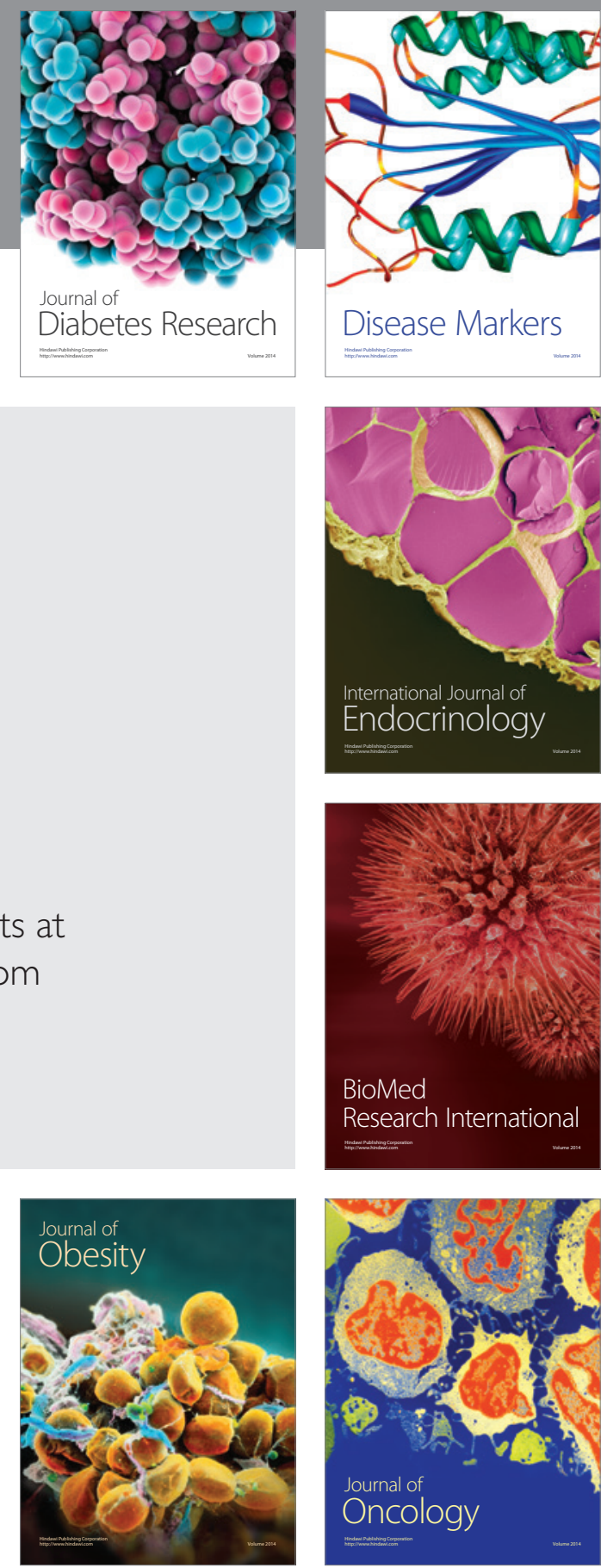

Disease Markers
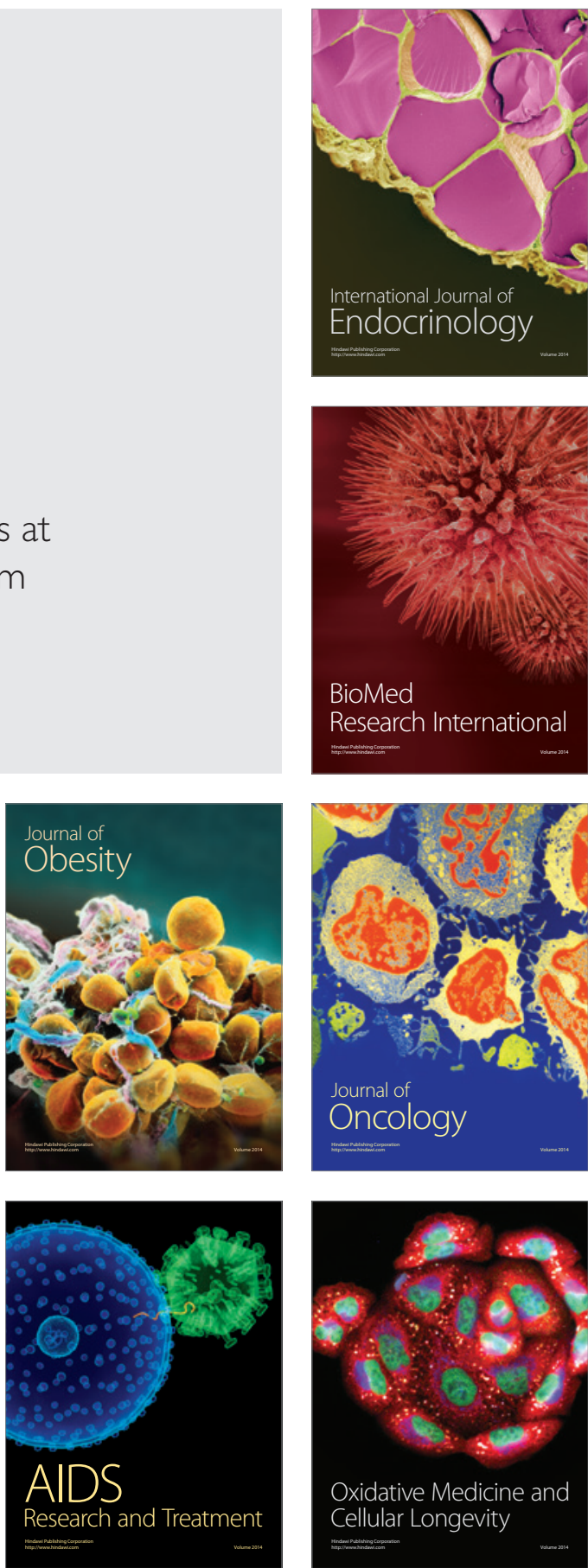